Indonesian Journal of Physics and NuclearApplications

Volume 3, Number 1, February 2018, p. 15-20

e-ISSN 2550-0570, (C) FSM UKSWPublication

\title{
Detail Engineering Design of Compact Neutron Generator to Support BNCT Facility in Indonesia
}

\author{
Djoko S. Pudjorahardjo, Widarto, Isman Mulyadi T \\ Center for Science and Technology of Accelerator - BATAN \\ Jl. Babarsari No.21, KotakPos 6101Ykbb, Yogyakarta 55281 \\ E-mail: s_pujoharjo@batan.go.id
}

\begin{abstract}
Boron Neutron Capture Therapy (BNCT) is a method of cancer therapy based on neutron radiation which has advantages over the other cancer therapy methods. It uses a stable isotope of 10B which will be an excited isotope of 11B when irradiated by thermal neutron. It immediately (in $10^{-12} \mathrm{~s}$ ) breaks into $\alpha$ particle and a lithium recoil nucleus. The two secondary particles play important roles in killing cancer cells. They have a short range in tissue $(5 \mu \mathrm{m}$ and 9 $\mu \mathrm{m}$ respectively) which is less than the average dimension of a cell. This leads to the damage of cancer cell only but the normal cells remain safe. Thermal and epithermal neutrons play important roles in BNCT. From the beginning the neutron sources for BNCT are nuclear reactors which produce high intensity of thermal neutrons (En $<0.5 \mathrm{eV}$ ), epithermal neutrons $(0.5 \mathrm{eV}<\mathrm{En}<10 \mathrm{keV})$ and fast neutrons $(\mathrm{En}>10 \mathrm{keV})$. However, nuclear reactors are very expensive and too large to be used in hospitals. In addition, the operation of nuclear reactors is under restricted protocols related to safety and physical protection. A compact neutron generator is a good choice of neutron source for BNCT. The advantages of compact neutron generator are that the size is small and that the neutron yield is more than $10^{9} \mathrm{~ns}^{-1}$ which satisfies the requirement recommended by IAEA. Additionally, the neutron energy is not so high that it requires a complicated neutron collimator, the operation is easy, and the public acceptance is higher than with nuclear reactors. Based on the requirements of epithermal neutron beam for BNCT facility, the detailed engineering design of compact neutron generator has been made.
\end{abstract}

Keywords Compact neutron generator, engineering design, BNCT

\section{INTRODUCTION}

An ideal therapy for cancer would be one whereby all tumor cells were selectively destroyed without damaging normal tissues. Most of the cancer cells should be destroyed, either by the treatment itself or with the help from the body's immune system; otherwise the danger exists that the tumor may reestablish itself. Although today's standard treatments (surgery, radiation therapy and chemotherapy) have successfully cured many kinds of cancers, there are still many treatment failures. The promise of a new experimental cancer therapy with some indication of its potential efficacy has led many scientists from around the world to work on an approach called boron neutron capture therapy (BNCT)[1].

BNCT is a surgically non-invasive, experimental radiation therapy developed for the treatment of malignant tumors of the brain. BNCT was first proposed by Locher in 1936. In $\mathrm{BNCT}$, a ${ }^{10} \mathrm{~B}$ containing compound called boron delivery agent is introduced into a patient. The patient is then irradiated in an epithermal neutron field. Neutrons from this field 
thermalize as they pass through brain tissue resulting in a thermal neutron flux at and around a tumor site. The ${ }^{10} \mathrm{~B}$ atoms, strongly absorb thermal neutrons in an $(n, \alpha)$ reaction and emit energetic $\alpha$ particles and ${ }^{7} \mathrm{Li}$ recoil nuclei that deposit their energy within approximately one cell diameter in the surrounding tissue ${ }^{[2]}$.

A wide variety of boron delivery agents have been synthesized, but only two of these currently are being used in clinical trials. The first, which has been used primarily in Japan, is a polyhedral borane anion, sodium borocaptate or BSA $\left(\mathrm{Na}_{2} \mathrm{~B}_{12} \mathrm{H}_{11} \mathrm{SH}\right)$, and the second is a dihydroxyboryl derivative of phenylalanine, referred to as boronophenylalanine or BPA. The latter has been used in clinical trials in the United States, Europe, Japan and more recently Argentina and Taiwan ${ }^{[3]}$.

Up until 1994, low energy thermal neutron beams (En $<0.5 \mathrm{eV}$ ) were used primarily in Japan, but since they have a limited depth of penetration in tissues, higher energy epithermal neutron beams $(0.5 \mathrm{eV}<$ En $<10 \mathrm{keV}$ ) which have a greater depth of penetration, have been used in clinical trials in United States, Europe and Japan ${ }^{[3]}$.

Clinical studies on BNCT originated at the Brookhaven National Laboratory (BNL) and the Massachusetts Institute of Technology (MIT) in collaboration with the Massachusetts General Hospital (MGH) approximately 60 years ago. The initial trials for the treatment high grade gliomas were carried out with thermal neutron beams produced by fission reactor. The neutrons energy spectra produced by fission reactor spanned from thermal neutrons $(\mathrm{En}<0.5 \mathrm{eV})$, epithermal neutrons $(0.5 \mathrm{eV}<\mathrm{En}<10 \mathrm{keV})$ and fast neutrons $(\mathrm{En}>10 \mathrm{keV})$. The clinical trials using epithermal neutrons were initiated at MIT and BNL in the early 1990's. These higher energy neutrons obviated the need for intraoperative BNCT when treating deep seated malignancies. Epithermal neutrons can reach tumor at the midline of the brain at a depth of $8 \mathrm{~cm}$ with therapeutic ratios $>1$. Epithermal neutrons are now generally used in BNCT irradiations although some intra-operative irradiations with thermal neutrons are still performed $^{[4]}$.

At present, BNCT is performed only at nuclear reactors, the unique facilities able to provide an intense epithermal and/or thermal neutron fluence rate to treat deep and/or shallow tumors. The treated pathologies are malignant brain tumors (glioblastoma multiforme), head and neck cancer, malignant (cutaneous and cerebral) melanoma and liver cancer. The promising results increased the interest in BNCT and encouraged the development of alternative neutron sources more suitable for in-hospital installation and of higher social acceptability than reactor. These sources could be operated in clinical environment, satisfy the clinical requests and increase the number of treated patients ${ }^{[5]}$.

Accelerator-based neutron sources are becoming an alternative choice to produce neutron beams for BNCT. The nuclear reaction ${ }^{7} \mathrm{Li}(\mathrm{p}, \mathrm{n})^{7} \mathrm{Be}$ seems to provide interesting results. However, the reaction requires high energy proton (1.8 - $4 \mathrm{MeV}$ ) available with expensive accelerating facilities which can hardly achieve beam current greater than few tens of $\mathrm{mA}$. Compact neutron generator $(\mathrm{CNG})$, based on the D-D or D-T fusion reaction are also investigated for BNCT purposes. The great advantage of $\mathrm{CNG}$ neutron sources is the low acceleration energy $(100-400 \mathrm{keV})$ of deuteron beam 
compared to that for the proton reaction ${ }^{7} \mathrm{Li}(\mathrm{p}, \mathrm{n}){ }^{7} \mathrm{Be}$, thus smaller accelerators with higher currents can be utilized ${ }^{[5]}$.

A compact neutron generator has been designed for supporting the BNCT facility in Indonesia. The activity was done in the frame of Consortium Incentive Research - National Innovation System Program titled Development of Technology and Application of Boron Neutron Capture Cancer Therapy Using Compact Neutron Generator. The program is coordinated by The Ministry of Research, Technology and Higher Education of Republic Indonesia.

\section{RESULTS AND DISCUSSION}

\subsection{Compact Neutron Generator}

Neutron generator is an ion accelerator used to produce fast neutrons based on D-D and D-T fusion reactions as follows:

${ }^{2} D+{ }^{2} D \rightarrow{ }^{1} n+{ }^{2} \mathrm{He}\left(E_{n}=2.45 \mathrm{MeV}, E_{3_{H e}}=0.82 \mathrm{MeV}\right)(2 a)$

${ }^{2} \mathrm{D}+{ }^{3} \mathrm{~T} \rightarrow{ }^{1} n+{ }^{4} \mathrm{He}\left(E_{n}=14.1 \mathrm{MeV}, E_{4} \mathrm{He}=3.5 \mathrm{MeV}\right)(2 \mathrm{~b})$

The energy levels of neutrons produced by each reaction are $2.45 \mathrm{MeV}$ and $14.1 \mathrm{MeV}$ respectively. The use of mono energetic neutrons produced by neutron generator for BNCT irradiations has advantages, i.e. the design and construction of beam shaping assembly (BSA) which is the component of BNCT which allows it to be simpler than if it were to use neutrons from nuclear reactor. Another advantage is that the energy of deuterons required to induce $\mathrm{D}-\mathrm{D}$ and $\mathrm{D}-\mathrm{T}$ fusion reactions is low enough $(100-400 \mathrm{keV})$. Therefore it only needs the low energy ion accelerator $^{[5]}$.

A compact neutron generator is a portable neutron generator developed for many fieldportable applications such as mine mineral mapping and analysis, petroleum exploration, explosives detection and identification, cement process control, coal quality analysis, nuclear waste assay, etc. These field applications need neutron generator which is of portable size and has a high neutron yield $\left(10^{8}-10^{11}\right.$ neutrons per second) as well as long-life operation ${ }^{[6]}$.

There are only a few producers of compact neutron generators in the world. One of these producers is The All-Russian Research Institute of Automatics (VNIIA) which produces three types of compact neutron generator i.e. vacuum tube-based neutron generator, gas-filled tubebased neutron generator and plasma focus based neutron generator. VNIIA serially produces neutron generators having flux up to $10^{10} \mathrm{n} / \mathrm{s}$. Urgent thermonuclear problem, radiotherapy; neutron radiography gave impetus for the development of neutron generators having $10^{10}$ $10^{11} \mathrm{n} / \mathrm{s}$ flux. The neutron generators are pictured in Fig. ${ }^{[7]}$. 

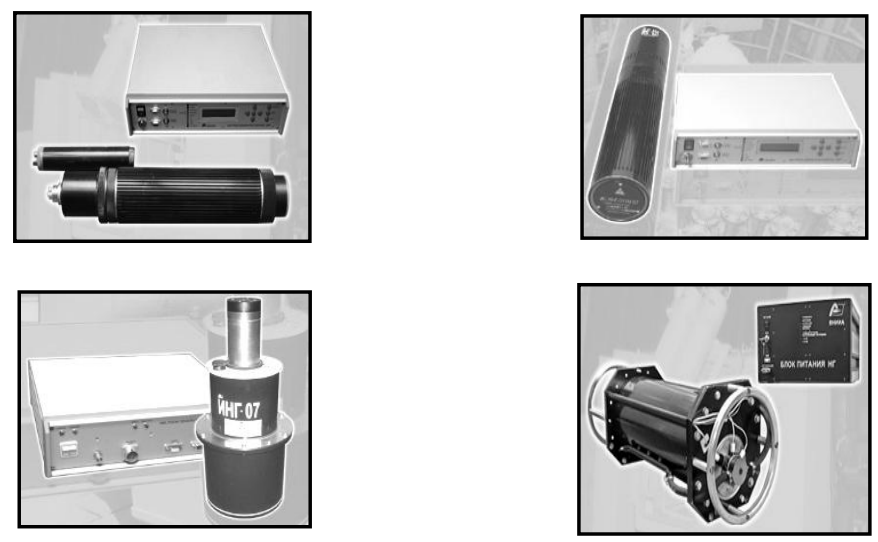

Figure 1. Compact neutron generators produced by VNIIA. The upper pictures are vacuum tube based neutron generators and the bottom pictures are gas-filled tube based neutron generators ${ }^{[7]}$.

\subsection{Description of Compact Neutron Generator Design}

The design of compact neutron generator for supporting BNCT facility is adapted from that by VNIIA produced gasfilled tube neutron generator. Typical circuit for gas-filled neutron tube is shown in Fig.2. A sealed neutron tube contains ion source, accelerating electrode system, and target combined in hermetic sealed housing. During operation at the heating getter the deuterium and tritium are released in tube volume and are ionized in the ion source ${ }^{[8]}$.

The ion source is a Penning type ion source which has long been used for neutrongeneration through D-D and D-T fusion reactions due to their simple design, low power consumption, ease of operation, durability and ability for compactness. This simple ion source consists of a hollow cylindrical anode (usually biased with 1 - $2 \mathrm{kV}$ ) with cathode plates at each end of the anode (usually at ground potential). An external magnet is arranged to generate a coaxial magnet field of several hundred gauss within the ion source. When deuterium and/or tritium gas is introduced into the anode at a pressure of a few mTorr, the electric field between the anode and cathodes ionizes the gas. Produced ions can escape the chamber into the acceleration section of the tube through a hole at the center of one of the cathodes ${ }^{[6,9]}$.

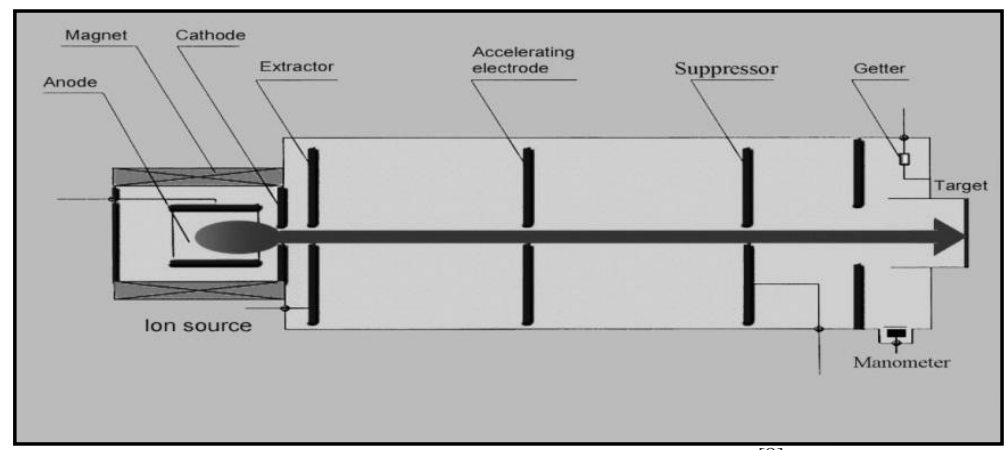

Figure 2.Gas-filled neutron tube circuit ${ }^{[8]}$.

The ions are formed into beams and accelerated between tube electrodes. There are 3 electrodes called extractor, accelerating electrode and suppressor. The extractor is used 
for extracting ions from the chamber and forming ion beams. The accelerating electrode then accelerates ion beams usually up to $\sim 110 \mathrm{kV}$. The suppressor (antidynatron electrode) provides suppression of secondary electron current from target. Accelerated ions bombard target saturated with deuterium and/or tritium, and neutrons are produced as a result of thermonuclear reactions ${ }^{[8]}$.

The neutron tube emits fast neutrons with a mean energy of $2.5 \mathrm{MeV}$ (D-D) or 14.1
$\mathrm{MeV}$ (D-T) which should be moderated to the required epithermal energy range $(1 \mathrm{eV}-10 \mathrm{keV})$ using suitable moderator materials. The ideal moderator must have a low scattering cross section to epithermal energies, high scattering cross section at higher energies without induced gamma rays from the interaction of the neutrons and the moderator material, a high absorption cross section in all energies except to the epithermal range. The values of neutron beam parameters for BNCT are recommended by IAEA as presented in Table $1^{[10]}$.

Table1. IAEA recommended values in the beam exit window ${ }^{[10]}$

\begin{tabular}{ll}
\hline BNCT Beam Port Parameters & Recommended Value \\
\hline Epithermal Neutron Flux & $\sim 10^{9} \mathrm{n} \cdot \mathrm{cm}^{-2} \cdot \mathrm{s}^{-1}$ \\
\hline Ratio of Fast Neutron Dose to Epithermal Neutron Flux & $<2.0 \times 10^{-13} \mathrm{~Gy} \cdot \mathrm{cm}^{2}$ \\
\hline Ratio of Gamma Dose to Epithermal Neutron Flux & $<2.0 \times 10^{-13} \mathrm{~Gy} \cdot \mathrm{cm}^{2}$ \\
\hline Ratio of Epithermal to Thermal Neutron Flux & $>100$ \\
\hline Ratio of Epithermal to Fast Neutron Flux & $>20$ \\
\hline Fast Energi Group & $\mathrm{E}>10 \mathrm{keV}$ \\
\hline Epithermal Energy Group & $1 \mathrm{eV} \leq \mathrm{E} \leq 10 \mathrm{keV}$ \\
\hline Thermal Energy Group & $\mathrm{E}<1 \mathrm{eV}$ \\
\hline
\end{tabular}

2.3 Detail Engineering Design of Compact Neutron Generator for BNCT Facility

Based on the description of a compact neutron generator and the requirements of neutron beams for BNCT, the detail engineering design of compact neutron generator for supporting BNCT facility has been made as below.

Table 2. Technical Specifications of Compact Neutron Generator

\begin{tabular}{ll}
\hline \multicolumn{1}{c}{ Parameters of CNG } & \multicolumn{1}{c}{ Technical Specifications } \\
\hline Operation mode & Continuous \\
\hline Neutron emission mode & Continuous (steady) \\
\hline Average neutron energy & $14 \mathrm{MeV}(\mathrm{D}, \mathrm{T}$ filled tube) \\
& $2.5 \mathrm{MeV}(\mathrm{D}$ filled tube $)$ \\
\hline Neutron output (flux) & $(0.5-1.0) \times 10^{11} \mathrm{n} / \mathrm{s}$ \\
\hline Tube life time & 300 hours \\
\hline Acceleration voltage & $(150-270) \mathrm{kV}$ \\
\hline Ion current & $(3-5) \mathrm{mA}$ \\
\hline Power supply & $220 \mathrm{~V},(50-60) \mathrm{Hz}$
\end{tabular}




\begin{tabular}{ll} 
Target & $\begin{array}{l}\text { Grounded, water cooled (water film thickness } \\
\text { near target } \sim 1 \mathrm{~mm} \text { ) }\end{array}$ \\
\hline Operation temperature & +15 to $+35^{\circ} \mathrm{C}$ \\
\hline Humidity & Up to $80 \%$ \\
\hline Power consumption & $\leq 1200 \mathrm{~W}$ \\
\hline Cooling system & - Use deionized water \\
& - Cooling power $1.8 \mathrm{~kW}$ \\
& - Temperature of coolant $-10 \pm 5^{\circ} \mathrm{C}$ \\
\hline
\end{tabular}

\section{CNG Engineering Design:}

1. Neutron Unit:

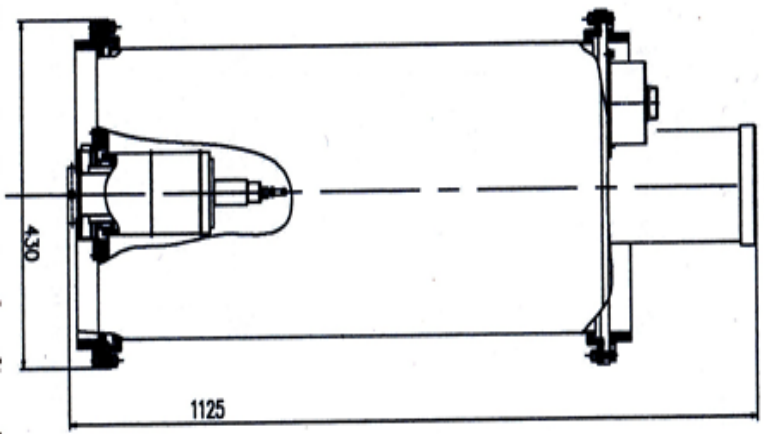

2. Neutron Tube:
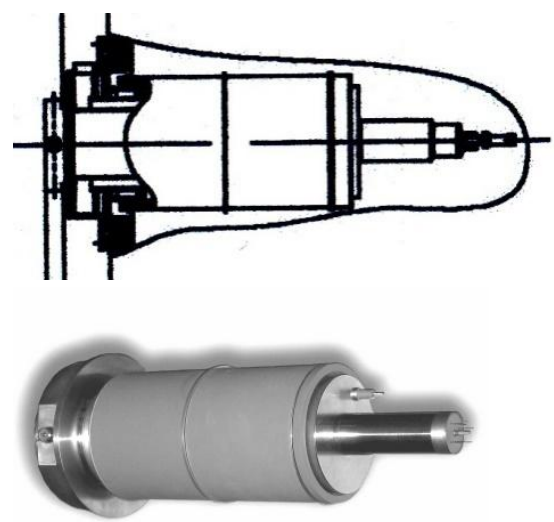

\section{Conclusion and Remarks}

The detail engineering design of compact neutron generator for BNCT facility has been made. The design is adapted from gas-filled neutron tube produced by VNIIA to fulfill the neutron beam requirements for BNCT facility.

\section{REFERENCES}

[1] The Basics of Boron Neutron Capture Therapy, http://web.mit.edu/nrl/www/ bnct/ info/description/description.html.

[2] Joshua P. Sroka, et al, Design of A Moderator Assembly Delimiter For An ABNS For BNCT, American Nuclear Society Topical Meeting in Monte Carlo, Chattanooga, Tennessee, April 17 21, 2005.

[3] Neutron Capture Therapy of Cancer, Wikipedia the free encyclopedia.

[4] Barth, R.F., et al, Current Status of Boron Neutron Capture Therapy of High Grade Gliomas and Recurrent Head and Neck Cancer. Radiation Oncology 7:146, 1-21, 2012.

[5] Durisi, E., et al, Design of an epithermal column for BNCT based on D-D fusion neutron facility. Nuclear Instruments and Methods in Physics Research A 574: 363 - 369, 2007.

[6] David L. Chichester and James D. Simpson, Compact Accelerator Neutron Generators, American Institute of Physics, December 2003/January 2004: 22-25.

[7] Neutron Generators, All-Russian Research Institute of Automatics (VNIIA), http://www.vniia.ru/eng/ng/element.html

[8] Yevgeni P. Bogolubov, et. al, VNIIA Neutron Generators for Thermonuclear Research.

[9] A. Sy, et. al, Novel Methods for Improvement of a Penning Ion Source for Neutron Generator Applications, Rev. Sci. Instrum. 83, 02B309, 2012.

[10] Fantidis, et. al, Optimised BNCT Facility Based on A Compact D-D Neutron Generator, Int. J. Radiat. Res., Vol.11 No.4, October, 2013. 